\title{
Green's Function Method for an Axisymmetric Void Between Parallel Walls
}

\author{
${ }^{*, \S}$ G. S. Chandekar, ${ }^{\dagger, \S}$ J. D. Richardson, ${ }^{\ddagger}$ Y. A. Melnikov, \\ and ${ }^{\S}$ S. J. Pardue \\ $\S$ Department of Mechanical Engineering; \\ Tennessee Technological University; Cookeville, TN 38505 USA \\ ${ }^{\ddagger}$ Department of Mathematical Sciences; \\ Middle Tennessee State University; Murfreesboro, TN 37132 USA
}

\begin{abstract}
The Green's function for potential theory is developed for an axisymmetric void of arbitrary shape located between two parallel walls. Numerical results are given to demonstrate the accuracy in the Green's function formulation by comparison with numerical solutions obtained using a commercial finite element code. The present formulation is attractive since numerical implementation only involves unknowns on the surface of the void.
\end{abstract}

\section{Introduction}

When possible, computational modeling of various physical problems can be significantly enhanced by the use of the associated Green's function. As such, the development of Green's functions continues to be of technical interest. For the case of potential theory arising from problems in heat conduction, several methods for obtaining exact expressions for Green's functions are described in [1]. The search for Green's functions may also be extended through semi-analytical approaches such as the method of variation of parameters [2,3].

Herein, consideration is given to the problem of voids of axisymmetric shape located between two parallel walls which are much larger than any characteristic dimension of the void. Within this level of generality, the Green's function presently to be developed may be applied to several physical problems including cavitation bubbles between walls, heat conduction between two plates where the conducting medium contains an axisymmetric void as well as consideration of the electrostatic field between two plates, for which the notion of a 'void' may be replaced with the insertion of perfect conductor of axisymmetric shape.

\footnotetext{
*Present address: Computational Science and Engineering; North Carolina A\&T State University; Greensboro, NC 27411, USA

${ }^{\dagger}$ Corresponding author: email: jrichardson@tntech.edu; Tele: 1931372 3197; FAX: 1931372 6340 .
} 
The following section outlines the development of the axisymmetric Green's function for an arbitrarily shaped void between two infinite walls. Subsequent sections will provide numerical results, showing strong agreement in the results obtained under the formulation presented here with those obtained from a domain discretization using finite element analyses (FEA) of heat conduction problems.

\section{Formulation of Green's Function for an Axisymmetric Void between Parallel Plates}

This section provides an overview of the development of the Green's function for potential theory for the general problem of axisymmetric voids between two infinite disks. The Green's function to be developed will be useful for a numerical approximation well suited for computational methods.

\subsection{Problem Description}

The problem to be considered is that of solving the Laplace's equation,

$$
\nabla^{2} \phi=0
$$

in the region $\mathscr{D}$, defined by $\mathscr{D}=\{0 \leq r<\infty, 0 \leq z \leq H\}$, between two parallel planes, where the Laplacian operator can be written in its axisymmetric form using cylindrical coordinates.

As a part of the development which will enable the consideration of arbitrarily shaped cavities [4], one may consider Poisson's equation given as,

$$
r \frac{\partial^{2} \phi}{\partial r^{2}}+\frac{\partial \phi}{\partial r}+r \frac{\partial^{2} \phi}{\partial z^{2}}=-r f(r, z)
$$

Under the assumptions of axisymmetry and boundedness, the mixed boundary value problem is given as that of finding $\phi$ such that,

$$
\begin{gathered}
\frac{\partial \phi(0, z)}{\partial r}=0, \quad|\phi(\infty, z)|<\infty, \\
\frac{\partial \phi(r, 0)}{\partial z}=0, \quad \phi(r, H)=0 .
\end{gathered}
$$

As explained in [2] if $G(r, z ; \rho, \zeta)$ represents the Green's function of homogeneous boundary value problem corresponding to that posed by Eq. (2) and Eqs. (3) and (4), then the unique solution of the problem can be expressed by the integral

$$
\phi(r, z)=\int_{0}^{H} \int_{0}^{\infty} G(r, z ; \rho, \zeta) f(\rho, \zeta) \rho \mathrm{d} \rho \mathrm{d} \zeta
$$

In order to develop an expression for the Green's function, the functions $\phi(r, z)$ and $f(r, z)$ may be expanded in a Fourier cosine series given as,

$$
\phi(r, z)=\sum_{n=1}^{\infty} \phi_{n}(r) \cos (\nu z)
$$


and

$$
f(r, z)=\sum_{n=1}^{\infty} f_{n}(r) \cos (\nu z)
$$

where

$$
\nu=\frac{(2 n-1) \pi}{2 H},
$$

chosen to satisfy the boundary conditions on $z$, and where $n$ is a positive, nonzero integer.

Upon substitution of these expansions in Eq. (2), the following problem is obtained,

$$
\sum_{n=1}^{\infty}\left(\left[r \frac{\mathrm{d}^{2}}{\mathrm{~d} r^{2}}+\frac{\mathrm{d}}{\mathrm{d} r}-r \nu^{2}\right] \phi_{n}(r)\right) \cos (\nu z)=-\sum_{n=1}^{\infty} r f_{n}(r) \cos (\nu z) .
$$

\subsection{Radial Component}

Equating each of the coefficients of $\cos (\nu z)$ in Eq. (9) one obtains,

$$
r \frac{\mathrm{d}^{2} \phi_{n}(r)}{\mathrm{d} r^{2}}+\frac{\mathrm{d} \phi_{n}(r)}{\mathrm{d} r}-r \nu^{2} \phi_{n}(r)=-r f_{n}(r)
$$

subject to the boundary conditions,

$$
\frac{\mathrm{d} \phi_{n}(0)}{\mathrm{d} r}=0, \quad\left|\phi_{n}(\infty)\right|<\infty .
$$

Following the method of variation of parameters [2, 3], a solution to Eq. (10) is sought of the form

$$
\phi_{n}(r)=C_{1}(r) I_{0}(\nu r)+C_{2}(r) K_{0}(\nu r),
$$

where $C_{1}(r)$ and $C_{2}(r)$ are differentiable functions to be determined and $\phi_{n, 1}(r) \equiv$ $I_{0}(\nu r)$ and $\phi_{n, 2}(r) \equiv K_{0}(\nu r)$ are the linearly independent solutions to the homogeneous form of Eq. (10). In order to constrain the system following the introduction of the pair of functions $C_{1}(r)$ and $C_{2}(r)$, Lagrange's assumption [2] is used,

$$
C_{1}^{\prime}(r) I_{0}(\nu r)+C_{2}^{\prime}(r) K_{0}(\nu r)=0,
$$

where the superscript prime is used to denote differentiation with respect to $r$.

Substitution of Eq. (12) into Eq. (10) along with Lagrange's assumption, gives the following expression,

$$
\begin{aligned}
& \left\{r I_{0}^{\prime \prime}(\nu r)+I_{0}^{\prime}(\nu r)-r \nu^{2} I_{0}(\nu r)\right\} C_{1}(r)+ \\
& \left\{r K_{0}^{\prime \prime}(\nu r)+K_{0}^{\prime}(\nu r)-r \nu^{2} K_{0}(\nu r)\right\} C_{2}(r) \\
& +C_{1}^{\prime}(r) I_{0}^{\prime}(\nu r)+C_{2}^{\prime}(r) K_{0}^{\prime}(\nu r)=-r f_{n}(r) .
\end{aligned}
$$


Since $I_{0}(\nu r)$ and $K_{0}(\nu r)$ represent solutions of the homogeneous equation associated with Eq. (10), the coefficients of $C_{1}(r)$ and $C_{2}(r)$ in the previous equation are zero. Hence,

$$
C_{1}^{\prime}(r) I_{0}^{\prime}(\nu r)+C_{2}^{\prime}(r) K_{0}^{\prime}(\nu r)=-r f_{n}(r) .
$$

Expressing Eq. (13) and Eq. (15) in matrix form yields the following system,

$$
\left[\begin{array}{ll}
I_{0}(\nu r) & K_{0}(\nu r) \\
I_{0}^{\prime}(\nu r) & K_{0}^{\prime}(\nu r)
\end{array}\right]\left\{\begin{array}{l}
C_{1}^{\prime} \\
C_{2}^{\prime}
\end{array}\right\}=\left\{\begin{array}{c}
0 \\
-r f_{n}(r)
\end{array}\right\} .
$$

Upon inversion, one obtains,

$$
\begin{aligned}
& C_{1}^{\prime}(r)=\frac{K_{0}(\nu r) r f_{n}(r)}{W(\nu r)}, \\
& C_{2}^{\prime}(r)=-\frac{I_{0}(\nu r) r f_{n}(r)}{W(\nu r)},
\end{aligned}
$$

where $W(\nu r)$ is used to denote the Wronskian of the homogeneous solution set,

$$
W(\nu r)=I_{0}(\nu r) K_{0}^{\prime}(\nu r)-K_{0}(\nu r) I_{0}^{\prime}(\nu r)
$$

Integrating $C_{1}^{\prime}(r)$ and $C_{2}^{\prime}(r)$, one obtains,

$$
\begin{aligned}
C_{1}(r) & =\int_{0}^{r} \frac{K_{0}(\nu \rho)}{W(\nu \rho)} \rho f_{n}(\rho) \mathrm{d} \rho+T_{1}, \\
C_{2}(r) & =-\int_{0}^{r} \frac{I_{0}(\nu \rho)}{W(\nu \rho)} \rho f_{n}(\rho) \mathrm{d} \rho+T_{2},
\end{aligned}
$$

where $T_{1}$ and $T_{2}$ are constants to be determined. Substitution of $C_{1}(r)$ and $C_{2}(r)$ into Eq. (12) gives,

$$
\begin{aligned}
\phi_{n}(r) & =\int_{0}^{r} \frac{K_{0}(\nu \rho) I_{0}(\nu r)-I_{0}(\nu \rho) K_{0}(\nu r)}{W(\nu \rho)} \rho f_{n}(\rho) \mathrm{d} \rho \\
& +T_{1} I_{0}(\nu r)+T_{2} K_{0}(\nu r) .
\end{aligned}
$$

Differentiating $\phi_{n}(r)$ in order to determine $T_{1}$ and $T_{2}$ yields,

$$
\begin{aligned}
\phi_{n}^{\prime}(r) & =\frac{\mathrm{d}}{\mathrm{d} r} \int_{0}^{r} \frac{K_{0}(\nu \rho) I_{0}(\nu r)-I_{0}(\nu \rho) K_{0}(\nu r)}{W(\nu \rho)} \rho f_{n}(\rho) \mathrm{d} \rho \\
& +T_{1} I_{0}^{\prime}(\nu r)+T_{2} K_{0}^{\prime}(\nu r) .
\end{aligned}
$$

The term associated with differentiation of the upper limit in accordance with Leibniz' theorem is zero for $\rho=r$.

Noting that the integral vanishes as $r \rightarrow 0$, the boundary condition at $r=0$ gives,

$$
\phi_{n}^{\prime}(0)=\lim _{r \rightarrow 0}\left(T_{1} I_{0}^{\prime}(r)+T_{2} K_{0}^{\prime}(r)\right)=0 .
$$


Since, as $r \rightarrow 0, I_{0}^{\prime}(r) \rightarrow 0$ and $K_{0}^{\prime}(r) \rightarrow \infty$, the term $T_{2}$ is seen to be zero.

From the requirement that $\phi_{n}(r)$ is bounded as $r \rightarrow \infty$,

$$
T_{1}=\lim _{r \rightarrow \infty}\left(\int_{0}^{r} \frac{I_{0}(\nu \rho) \frac{K_{0}(\nu r)}{I_{0}(\nu r)}-K_{0}(\nu \rho)}{W(\nu \rho)} \rho f_{n}(\rho) \mathrm{d} \rho\right) .
$$

Since, as $r \rightarrow \infty, I_{0}(r) \rightarrow \infty$ and $K_{0}(r) \rightarrow 0$, the fraction in the left side of the numerator of the integrand is zero leaving,

$$
T_{1}=-\int_{0}^{\infty} \frac{K_{0}(\nu \rho)}{W(\nu \rho)} \rho f_{n}(\rho) \mathrm{d} \rho .
$$

Following the determination of $T_{1}$ and $T_{2}$, Eq. (21) is written as,

$$
\begin{aligned}
\phi_{n}(r) & =\int_{0}^{r} \frac{K_{0}(\nu \rho) I_{0}(\nu r)-I_{0}(\nu \rho) K_{0}(\nu r)}{W(\nu \rho)} \rho f_{n}(\rho) \mathrm{d} \rho \\
& -\int_{0}^{\infty} \frac{K_{0}(\nu \rho) I_{0}(\nu r)}{W(\nu \rho)} \rho f_{n}(\rho) \mathrm{d} \rho .
\end{aligned}
$$

Separating the second integral as,

$$
\begin{aligned}
& \int_{0}^{\infty} \frac{K_{0}(\nu \rho) I_{0}(\nu r)}{W(\nu \rho)} \rho f_{n}(\rho) \mathrm{d} \rho= \\
& \int_{0}^{r} \frac{K_{0}(\nu \rho) I_{0}(\nu r)}{W(\nu \rho)} \rho f_{n}(\rho) d \rho+\int_{r}^{\infty} \frac{K_{0}(\nu \rho) I_{0}(\nu r)}{W(\nu \rho)} \rho f_{n}(\rho) \mathrm{d} \rho
\end{aligned}
$$

and combining integrals with identical limits one obtains,

$$
\phi_{n}(r)=-\int_{0}^{r} \frac{I_{0}(\nu \rho) K_{0}(\nu r)}{W(\nu \rho)} \rho f_{n}(\rho) \mathrm{d} \rho-\int_{r}^{\infty} \frac{K_{0}(\nu \rho) I_{0}(\nu r)}{W(\nu \rho)} \rho f_{n}(\rho) \mathrm{d} \rho .
$$

This finally yields,

$$
\phi_{n}(r)=\int_{0}^{\infty} g_{n}(r, \rho) f_{n}(\rho) \rho \mathrm{d} \rho,
$$

where the kernel $g_{n}(r, \rho)$ is defined as

$$
g_{n}(r, \rho)= \begin{cases}\frac{-K_{0}(\nu \rho) I_{0}(\nu r)}{W(\nu \rho)}, & \text { for } r \leq \rho \\ \frac{-I_{0}(\nu \rho) K_{0}(\nu r)}{W(\nu \rho)}, & \text { for } \rho \leq r\end{cases}
$$

\subsection{Axisymmetric Green's Function for the Infinite Disk}

Following the determination of the radial components $\phi_{n}(r)$, the solution for Eq. (10) is sought. Writing Eq. (7) in terms of $\rho$ and $\zeta$ and substituting for each $f_{n}$ using,

$$
f_{n}(\rho)=\frac{2}{H} \int_{0}^{H} f(\rho, \zeta) \cos (\nu \zeta) \mathrm{d} \zeta
$$


one obtains,

$$
\phi_{n}(r)=\int_{0}^{H} \int_{0}^{\infty} \frac{2}{H} g_{n}(r, \rho) \cos (\nu \zeta) f(\rho, \zeta) \rho \mathrm{d} \rho \mathrm{d} \zeta
$$

Upon substitution into Eq. (6) and exchanging the order of summation and integration,

$$
\phi(r, z)=\int_{0}^{H} \int_{0}^{\infty} G(r, z ; \rho, \zeta) f(\rho, \zeta) \rho \mathrm{d} \rho \mathrm{d} \zeta
$$

where the kernel $G(r, z ; \rho, \zeta)$ is the Green's function given as,

$$
G(r, z ; \rho, \zeta)=\sum_{n=1}^{\infty} \frac{2}{H} g_{n}(r, \rho) \cos (\nu \zeta) \cos (\nu z)
$$

Finally, for the case that the source term $f(\rho, \zeta)$ is taken to represent a density $\lambda(\rho, \zeta)$ on a surface $\Gamma$, the following integral representation is obtained,

$$
\phi(r, z)=\int_{\Gamma} G(r, z ; \rho, \zeta) \lambda(\rho, \zeta) \mathrm{d} \Gamma(\rho, \zeta)
$$

As will be shown in the next section, for the case of a known potential on an arbitrary void, the resulting first kind integral equation may be readily solved on a fictitious contour which is slightly offset from the given geometry using the method of functional equations [5], also often referred to as the method of fundamental solutions. The numerical results to be presented will demonstrate that the Green's function developed here in the form given by Eqs. (30) and (34) is directly suitable for numerical computation.

\section{$3 \quad$ Numerical Results}

This section presents some numerical results which demonstrate the usefulness and accuracy in the current method. The numerical implementation of the method is based on Fortran routines for the evaluation of the Green's function along with the adaptation of a previously existing Fortran boundary element code [6] to build and solve the linear system developed from Eq. (35).

\subsection{Point Source Solution}

The expression in Eq. (33) is used to evaluate the numerical accuracy in the Green's function under the particular consideration that the function $f(\rho, \zeta)$ represents a line source corresponding to a Dirac function. In such case, the solution $\phi(r, z)$ reduces to the Green's function evaluated between the two points $(r, z)$ and $(\rho, \zeta)$. The following numerical examples are based on disks defined by $0 \leq z \leq 5$.

The solution may be compared with an approximate solution of a similar problem using a commercial FEA code. As a matter of convenience for use with the FEA, 


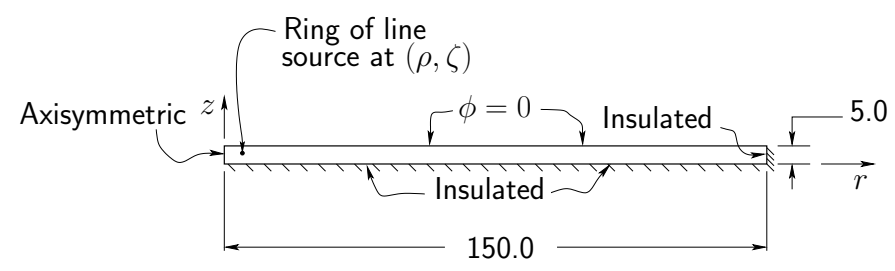

Figure 1: FEA problem geometry in the finite domain.

the Green's function may be considered in the context of heat conduction as shown in Figure 1 where the source term may be interpreted as a heat source ring. As shown in the figure, the infinite domain is modeled in the FEA by using an insulated condition at some large value of $r / H$ which is taken as 30 under the assumption that the radial component of the (heat) flux is negligible for $r>30 H$. In order to be consistent with this approximation, the ring of line source was placed close to the symmetry axis.

For the case of source ring placement at $(2,1)$ the solution obtained from the Green's function is shown in Figure 2 along with a finite element solution using approximately $6.8 \times 10^{4}$ nodes. The large number of nodes which was used in the FEA was needed in order to obtain sufficient smoothness in the contours very close to the ring source which will be shown subsequently. The contour plots in Figure 2 are based on a regular grid of points from the Green's function and the irregularly spaced nodes from the FEA. The figure only shows labeling for a limited number of contours; the remaining contours are each uniformly separated by 0.1 . For the contours shown, the two plots are visibly indistinguishable when superposed. The contours very near to the source grid are not shown for the Green's function in Figure 2 since they were affected by the coarseness of the grid sampling in that region; the near-field behavior of the Green's function is also reserved for a subsequent plot.

For the near-field behavior, contours are shown in Figure 3 for this example using both 80 terms in the cosine series and 200 terms. The contours from the Green's function shown in Figure 3 differ from the previous plots in that a radial search method was implemented to find the points on the contour in one-degree increments with a tolerance on the potential of $10^{-6}$. The plots differ primarily in that the contours from the Green's function with 80 terms are elongated in the $z$-direction in comparison to the contours from the 200 term expansion. Both plots show that the numerical Green's function has a discontinuity in slope at $r=\rho$.

When 500 terms are taken in the series, the previous contours of the Green's function become more nearly circular and compare very closely with contours taken from an FEA solution as shown in Figure 4. Though still present, the size of the cusp in the Green's function contours is considerably reduced in comparison with the 200 term solution.

While the discontinuity in slope of the Green's function is visually striking, 


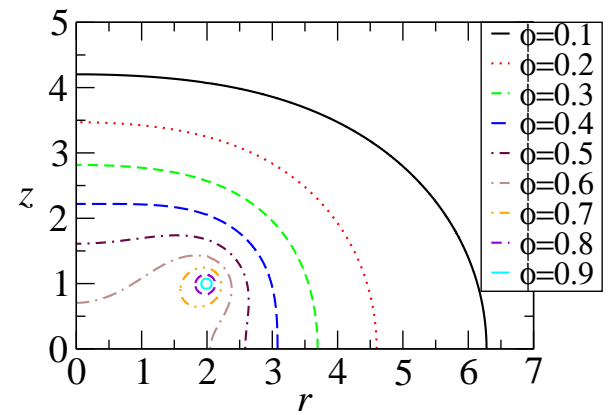

(a) Green's function (80 terms)

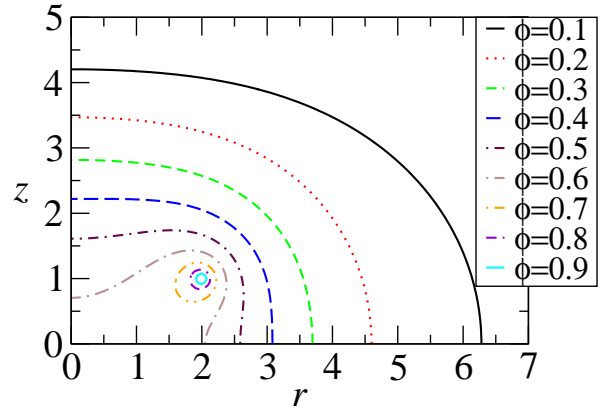

(b) FEA solution

Figure 2: Uniformly spaced contour lines of $\phi$ for source ring placement at $(\rho, \zeta)=$ $(2,1)$.

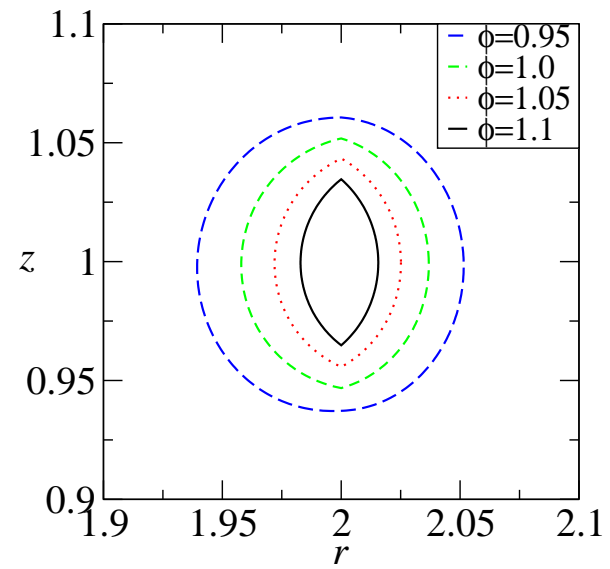

(a) 80 terms

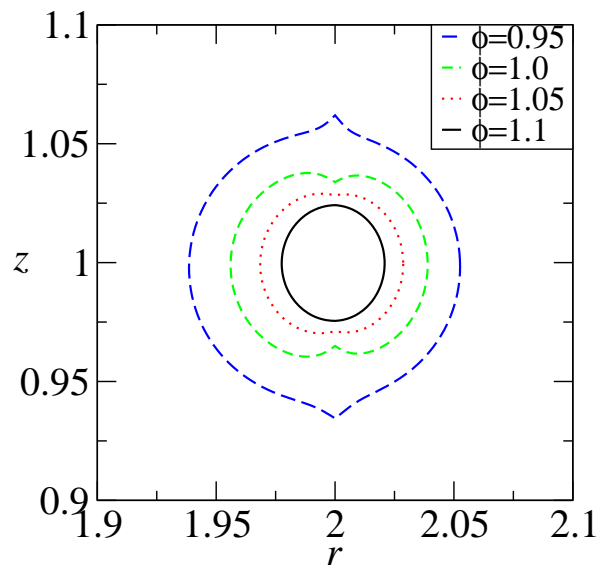

(b) 200 terms

Figure 3: Numerical behavior of contour points in the near-field for $(\rho, \zeta)=(2,1)$. 
each contour represents a difference in the value of the Green's function of approximately $5 \%$ in comparison with adjacent contours so that the accuracy in the Green's function is still high even for $r=\rho$. Away from the line $r=\rho$, accuracy in the contours is high for both the 200 and 500 term approximations. It is also noted that, for the desired problem of modeling arbitrary voids, the Green's function will be integrated over a void surface which will somewhat mitigate the isolated band of small inaccuracy for $r$ near $\rho$. The following section will show that voids of arbitrary shape may be modeled with a very high level of accuracy with the present approach.

\subsection{Numerical Results for an Axisymmetric Void}

In this section, the integral equation formulation is extended to the domain shown in Figure 5 which contains a void near the lower wall. The void geometry shown can be considered to be similar to a collapsing cavitation bubble near a rigid wall [7]. The void contour is partially dimensioned in order to provide a sense of its proximity to the lower surface. The boundary condition prescribed on the surface of the void is $\phi=1$ as shown in the figure. All numerical results presented in this section involving contours taken from the Green's function approach, unless stated otherwise, are based on a discretization of the void's surface using six sextic boundary elements [6], resulting in a model having only 37 degrees of freedom.

For the present case, both the integration order and the number of terms taken in the series are based on proximity of the source point and field point. The basis for the selection of the integration order and the number of terms was informally taken from comparison of the boundary solutions with those obtained from using a 96 -point quadrature scheme and 500 terms in the series everywhere. Defining a characteristic element length $\ell$ as the distance between the first and last nodes of the first element and $\left|\mathbf{r}_{P, Q}\right|$ as the distance between the source and field points, the quadrature scheme was set at 64-point when $\left|\mathbf{r}_{P, Q}\right| \leq 0.7 \times \ell$, 20-point when $0.7 \times \ell<\left|\mathbf{r}_{P, Q}\right| \leq 1.2 \times \ell$ and 12-point otherwise. The number of terms used in the cosine series was based on $|r-\rho|$ according to 500 terms used when $|r-\rho| \leq 0.03,200$ terms used when $0.03<|r-\rho| \leq 0.1$ and 150 terms used otherwise. When this spatially dependent scheme was employed, the average nodal difference between the boundary solutions from the spatially dependent scheme and the aforementioned 96-point, 500 term scheme only differed by approximately $1.46 \times 10^{-3}$ when normalized by the maximum nodal value of the density which was approximately 2.79 . The slight difference in the boundary solutions informally suggests the suitability of the spatially dependent scheme which obviously offers some reduction in computational expense.

As previously discussed, the integral equation based on Eq. (35) is solved for this problem on a fictitious contour which is slightly interior to the void as shown in Figure 6. The contour offset which was used for the numerical results to be presented was arbitrarily chosen as 0.25 times the distance between the fourth and fifth nodes of the lowest numbered element which included the source node. The offset direction was taken as the outward normal to the domain as computed 


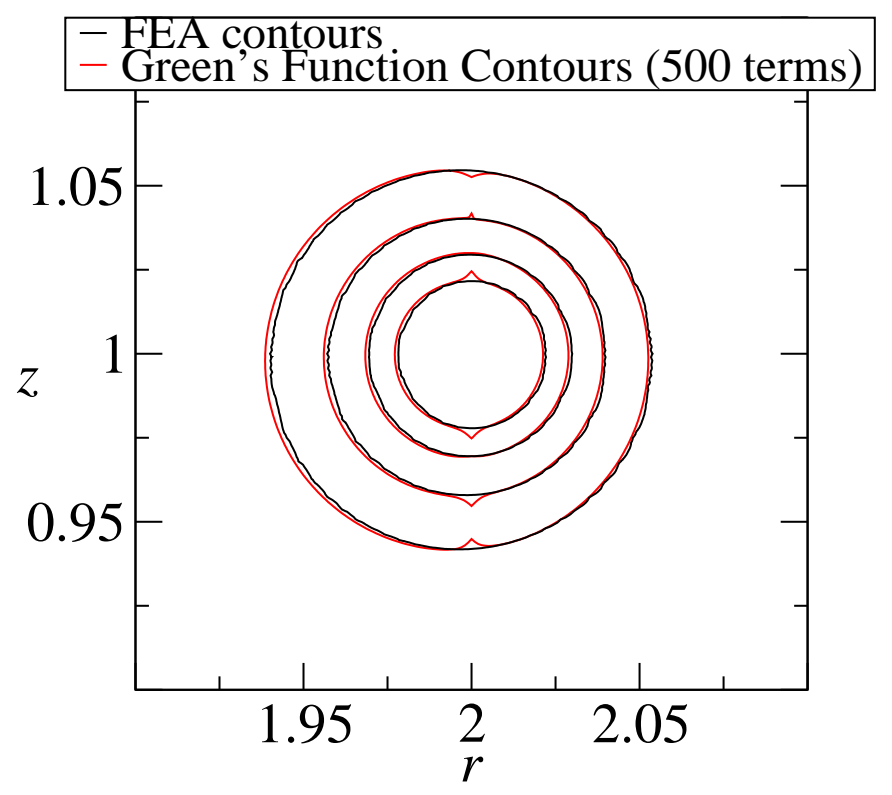

Figure 4: Comparison of the numerical Green's function with 500 terms with an FEA solution (contours of $\phi=0.95,1.0,1.05$ and 1.1).

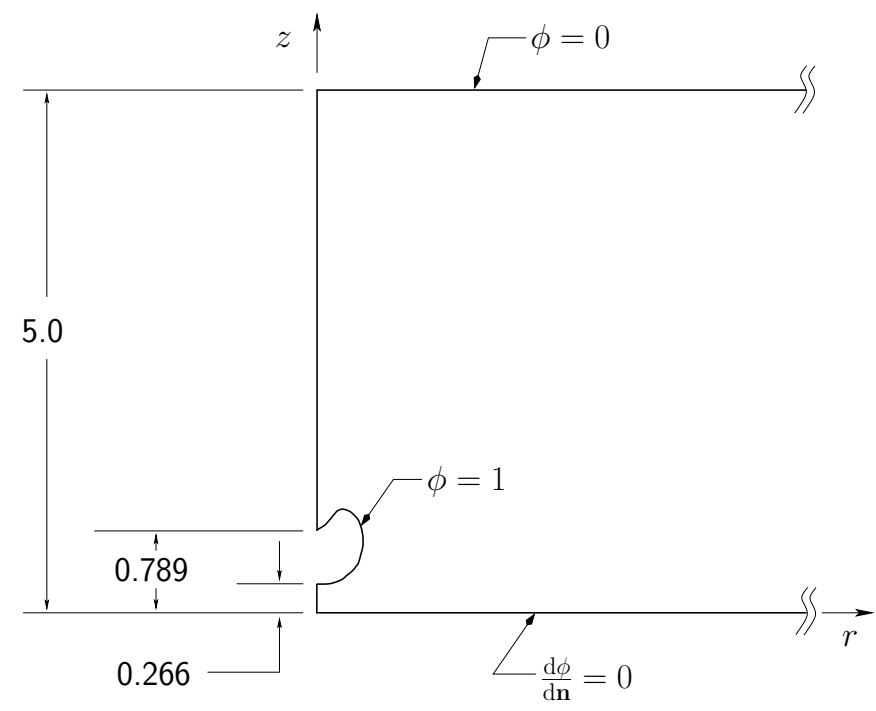

Figure 5: Axisymmetric void in the space between infinite disks. 


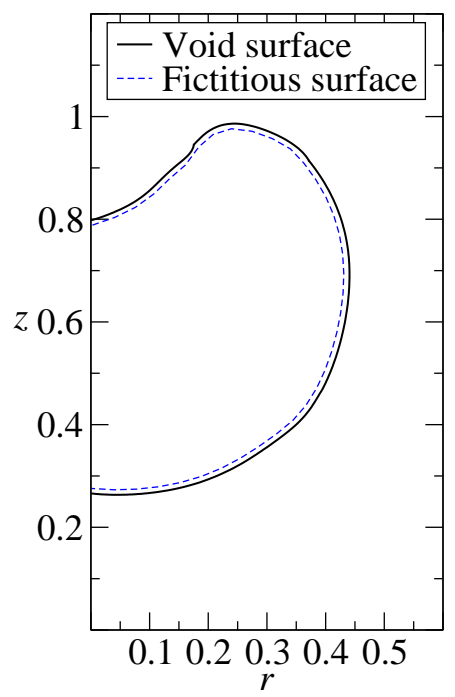

Figure 6: Fictitious contour used for computing void surface density; offset distance is $0.25 \times$ the interior nodal separation distance.

directly by differentiation of the shape functions. While limited numerical experimentation was performed regarding the amount of offset, the problem was also solved using an offset of 0.35 times the previously mentioned distance between nodes. The average absolute difference in the computed boundary data in the two cases was approximately $5.27 \times 10^{-3}$ when normalized by the aforementioned maximum value of the fictitious density for the nearer contour of approximately 2.79. Informal confidence in the suitability of the chosen contour was based on proximity of the contour to the void's surface as seen from Figure 6 and the fact that, when the contour separation is increased rather slightly, the computed boundary data is also only slightly changed.

The convergence behavior in the boundary solution may be observed from Figure 7 which shows the boundary solutions from the aforementioned six-element model as well as from an eight-element model plotted against a normalized surface intrinsic coordinate which starts on the upper portion of the void's surface. The offset of the fictitious contour in the eight-element model was adjusted to match that of the six-element model for consistency. The plot indicates that the two models show significant agreement, including a similar trend through some peaks in the upper part of the curve.

Demonstrated accuracy in the numerical Green's function formulation, again, may be inferred from the comparison of its interior solution with solutions of the similar finite domain problem obtained from a commercial FEA code. Following the determination of the boundary data on the void, a file of data points was created for post-processing of the Green's function interior point calculations. Contour plots of the potential at these data points from the two methods are given 


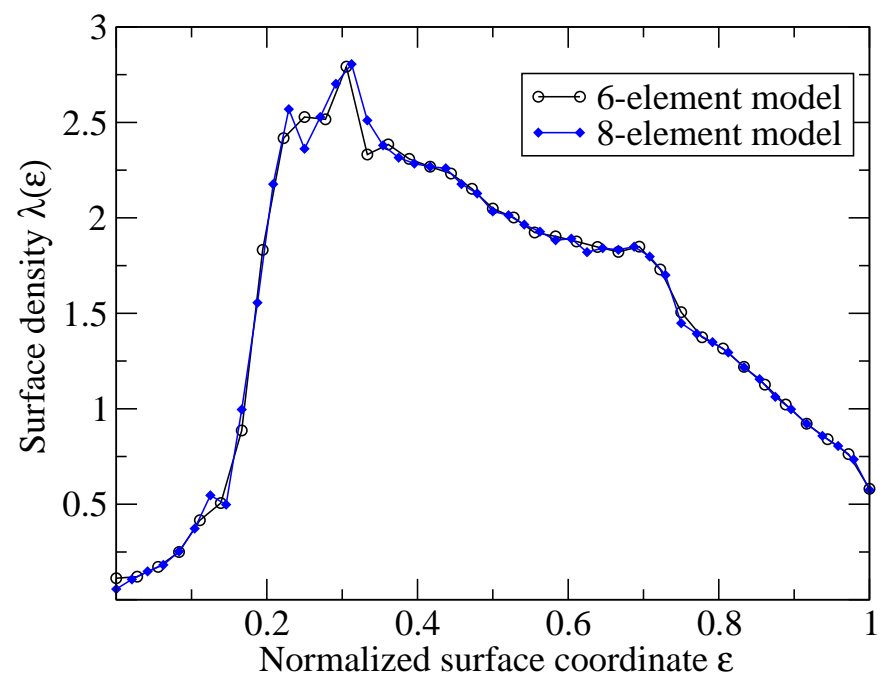

Figure 7: Boundary solutions on the void from different meshes.

in Figure 8. Close examination of the figure shows very strong agreement in the sets of contours taken from the two different methods.

For the near-void region, a gradient search algorithm was developed for the Green's function post-processor to find contours based on a tolerance of $10^{-6}$. This algorithm was used to find the contours from 0.91 to 0.99 near the void as shown in Figure 9. The contours are seen to be very smooth and are consistent with a potential of $\phi=1$ on the void's surface.

The present case cannot claim to provide an exhaustive comparative study between the computational efficiencies of the Green's function method and the FEA since the domain method should be expected to have significant dependence on geometric parameters including the disk height $H$ and the radius at which an insulated edge condition may serve as an approximation. Additionally, discretization levels in the two methods have not been studied extensively nor has kernel sampling [8] been explored with the Green's function method. In spite of these limitations, some computational comparisons between the Green's function method and the FEA may be given. Numerical experiments for both the FEA and the Green's function method were run on a single Pentium 4 Prescott $(3 \mathrm{GHz})$ processor on a machine with $1 \mathrm{~Gb}$ of RAM running Debian GNU/Linux 4.0. The g77 version 3.4.6 Fortran compiler was used for the Green's function method. The FEA model considered in this comparison had approximately $1.06 \times 10^{4}$ linear elements and $1.11 \times 10^{4}$ nodes in a highly graded mesh while the boundary discretization for the Green's function method had 37 nodes and six sextic elements as previously noted. The discretization chosen for the FEA used 36 linear elements around the surface of the void, also leading to 37 nodes on the void. Qualitatively, the FEA mesh could be considered as sufficiently refined to the conservative level of accept- 


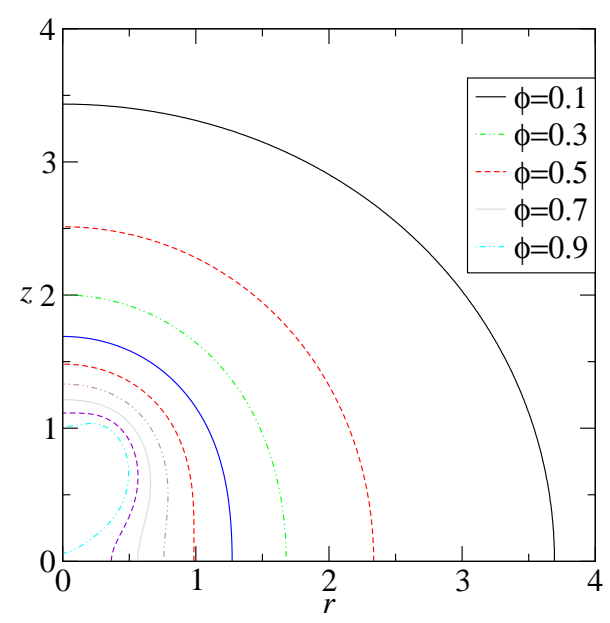

(a) Green's function

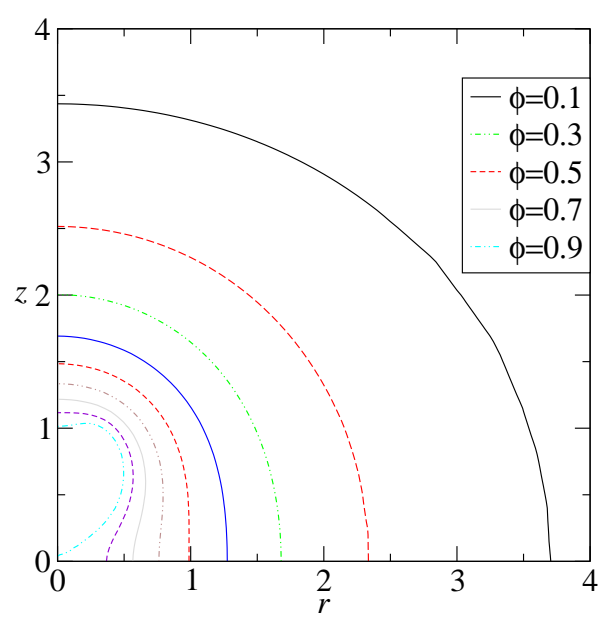

(b) FEA Solution

Figure 8: Lines of constant $\phi$ with uniform contour spacing of 0.1 .

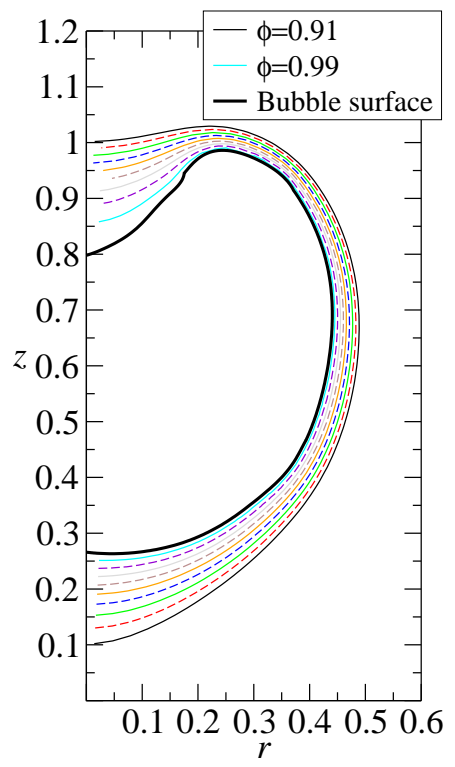

Figure 9: Uniform contours on intervals of 0.01 from $\phi=0.91$ to $\phi=0.99$ as determined from the numerical Green's function (500 terms). 
ability as some artifacts of the discretization were mildly detectable in the derived flux quantities after nodal averaging. The total run time for the commercial FEA was approximately 14.1 CPU seconds of which approximately 12.3 seconds was devoted to meshing of the domain. For comparison, the Green's function method offered a slight computational savings, taking approximately $10.6 \mathrm{CPU}$ seconds for the solution with negligible meshing.

\section{Conclusions}

A numerical Green's function was developed to model axisymmetric voids of arbitrary shape within the infinite disk which should be useful to model several types of axisymmetric physical problems in potential theory including cavitation bubbles, heat conduction and electrostatics. The present Green's function approach offers key computational advantages over alternative approaches. In addition to the usual reduction in meshing encumbrance which integral equations offer over domain methods, the present approach also removes the need to mesh surfaces other than that of the void. In the example considered, the solution of the Green's function method took less time, approximately 10.6 CPU seconds, than it took to mesh the domain, approximately $12.3 \mathrm{CPU}$ seconds, for a commercial FEA package. Additionally memory requirements to accurately model problems can be quite small for the Green's function method; numerical results based on a model having only 37 nodes showed excellent agreement with FEA solutions based on an $\mathcal{O}\left(10^{4}\right)$ node model of a similar problem solved in a large but finite domain.

\section{References}

[1] J. V. Beck, K. D. Cole, A. Haji-Sheikh, and B. Litkouhi. Heat Conduction Using Green's Functions. Hemisphere, Washington, DC, 1992.

[2] Yuri A. Melnikov. Influence Functions and Matrices. Marcel Dekker, New York, 1999.

[3] Abdul J. Jerri. Introduction to Integral Equations With Applications. John Wiley and Sons, Inc., New York, 1999.

[4] Y. A. Melnikov. Influence functions of a point source for perforated compound plates with facial convection. Journal of Engineering Mathematics, 49(3), pp. 253-270, 2004.

[5] V. D. Kupradze. Potential Method in the Theory of Elasticity. Daniel Davey, New York, 1965.

[6] S. Arjunon and J. D. Richardson. Regularized p-version collocation BEM algorithms for two-dimensional heat conduction. Engineering Analysis with Boundary Elements, 29, pp. 953-962, 2005. 
G.S. Chandekar et al. / Electronic Journal of Boundary Elements, Vol. 5, No. 2, pp. 69-83 (2007)

[7] W. Lauterborn and H. Bolle. Experimental investigations of cavitation-bubble collapse in the neighbourhood of a solid boundary. Journal of Fluid Mechanics, 72, pp. 391-399, 1975.

[8] J. D. Richardson, L. J. Gray, T. Kaplan, and J. A. L. Napier. A regularized spectral BEM for plane elasticity. Engineering Analysis with Boundary Elements, 25, pp. 297-311, 2001. 